\title{
Conceptos modernos de optimación termodinámica en centrales termoeléctricas mexicanas
}

\author{
J. Cervantes-de Gortari, A.Vidal-Santo, F. Méndez-Lavielle y O. Bautista-Godínez \\ Departamento de Termoenergía. División de Ingeniería Mecánica e Industrial \\ Facultad de Ingeniería, UNAM \\ E-mail:jgonzalo@servidor.unam.mx
}

(recibido: julio de 2001; aceptado: octubre de 2001)

\begin{abstract}
Resumen
En este trabajo se revisa el análisis de máxima generación de potencia que implica la minimización de la producción de entropía en centrales generadoras de potencia, de acuerdo con los lineamientos más recientes publicados en la literatura. Los conceptos y resultados del análisis se aplican a varias centrales mexicanas y se comparan con otras centrales en el mundo.
\end{abstract}

Descriptores: optimación termodinámica, centrales termoeléctricas, segunda ley.

\section{Abstract}

In this paper, a summary review of the current thermodynamic concepts related to the maximum power generation and minimum entropy production are presented. The results of the analysis are considered in Mexican power stations using conventional cycles and are compared with other power stations around the world.

Keywords: thermodynamic optimization, power plants, second law.

\section{Introducción}

El crecimiento actual de la demanda de energía eléctrica en México se ubica alrededor del $6 \%$ anual, lo que implica márgenes de reserva más bajos que en los últimos veinte años. Debido a esto, se han abierto líneas de investigación y desarrollo con objeto de buscar y analizar tecnologías que permitan las mejores eficiencias en la generación de potencia, tanto en centrales existentes como en las proyectadas.

La tendencia observada en el estudio termodinámico de los ciclos de potencia y sus aplicaciones durante los últimos veinte años, señala un interés renovado en el análisis de diversos aspectos relacionados con la Segunda Ley. De manera muy breve, se puede decir que la ingeniería térmica en este campo se ha combinado con las disciplinas de transferencia de calor y de mecánica de fluidos, para ofrecernos el modelado y la optimación de operaciones irreversibles caracterizadas por dispositivos de tamaños finitos, que emplean tiempos finitos. Existe una amplia literatura al respecto (Bejan et al., 1996 y Bejan, 1996a), que incluye tópicos y conceptos aceptados en la práctica, como el análisis exérgico, la termoeconomía y la minimización de generación de entropía, así como otros con menos aceptación 
general como el de termodinámica de tiempos finitos (o termodinámica endorreversible).

En el caso de las centrales termoeléctricas se trata de modelar su operación inherentemente irreversible, en términos de los componentes de transferencia de calor más simples, buscando identificar las oportunidades termodinámicas de compromiso para ajustar las características físicas del sistema que permitan su operación con la mínima generación de entropía.

Ha sido demostrado de manera amplia (Bejan, 1996b), que minimizar la generación de entropía en una central termoeléctrica es equivalente a maximizar su potencia de entrega. Para ello, es necesario considerar diversos aspectos de la irreversibilidad asociada a la transferencia de calor, especialmente la de los equipos de tamaño finito que se presentan en una central termoeléctrica.

En este trabajo se resume el análisis de máxima generación de potencia que implica la minimización de la producción de entropía en el ciclo de centrales generadoras de potencia, de acuerdo con los lineamientos más recientes publicados en la literatura. Los conceptos y resultados del análisis se aplican a varias centrales mexicanas y se comparan con otras centrales.

\section{Modelo simple del ciclo de una central termoeléctrica}

Existen diversos modelos de una máquina irreversible de calor que se han considerado para la optimación termodinámica del ciclo de una central termoeléctrica, ampliamente documentados en la literatura (p.ej., Bejan, 1996a). En ellos, se proponen diferentes alternativas para los intercambiadores de calor que operan en ambos extremos de temperatura del ciclo de Rankine, desde los más sencillos esquemas de Novikov (1958) y de Chambadal (1958), con un solo intercambiador en el extremo de alta temperatura, hasta el afamado modelo de Curzon y Ahlborn (1975), que toma en cuenta sendos intercambiadores en los extremos de temperatura del ciclo.

El modelo que se utiliza en el presente trabajo, ha sido concebido y desarrollado enteramente por
Bejan (1996a) y se basa en la consideración de que adicionalmente a las irreversibilidades externas al ciclo mismo, asociadas a la transferencia de calor en los saltos finitos de temperatura en los dos intercambiadores de calor, se tienen irreversibilidades provocadas, por lo que podría denominarse transferencia de calor interna, que se transmite directamente a través de las paredes del generador de vapor o del horno, de válvulas y conexiones, etc. La transferencia de calor interna, identificada por primera vez como una causa de irreversibilidad de la central por Bejan y Paynter (1976), constituye una derivación en paralelo a la transferencia de calor, a través de los intercambiadores de calor, como se ilustra esquemáticamente en la figura 1 .

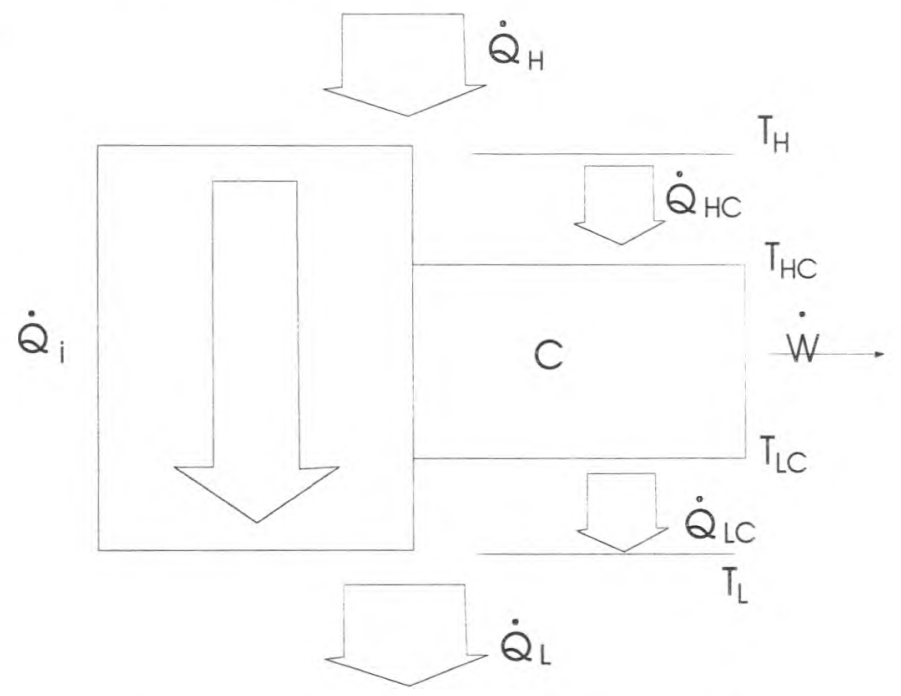

Figura 1. Modelo de central de potencia con transferencia de calor interna, de acuerdo con Bejan (1996 a)

Se puede escribir así, para las tres fuentes de irreversibilidad en la central:.

$$
\begin{gathered}
\dot{\mathrm{Q}}_{i}=\frac{\mathrm{T}_{\mathrm{H}}-\mathrm{T}_{\mathrm{L}}}{\mathrm{R}_{i}} \\
\dot{\mathrm{Q}}_{\mathrm{HC}}=(\mathrm{UA})_{\mathrm{H}}\left(\mathrm{T}_{\mathrm{H}}-\mathrm{T}_{\mathrm{HC}}\right) \\
\dot{\mathrm{Q}}_{\mathrm{LC}}=(\mathrm{UA})_{\mathrm{L}}\left(\mathrm{T}_{\mathrm{LC}}-\mathrm{T}_{\mathrm{L}}\right)
\end{gathered}
$$

donde $R_{i}$ es la resistencia térmica interna, $(U A)_{H} y$ (UA) L son las conductancias térmicas de los 
intercambiadores de calor en los extremos caliente y frío respectivamente; los demás símbolos corresponden a los parámetros y variables señalados en la figura 1. De acuerdo con el procedimiento de Bejan (1996a), se define la conductancia térmica total de ambos intercambiadores,

$$
\mathrm{UA}=(\mathrm{UA})_{\mathrm{H}}+(\mathrm{UA})_{\mathrm{L}}
$$

así como la razón de distribución de conductancias $x$,

$$
\chi=\frac{(\mathrm{UA})_{\mathrm{H}}}{\mathrm{UA}}
$$

que sustituidas en la ecuación 3 da como resultado:

$$
\dot{\mathrm{Q}}_{\mathrm{LC}}=(1-x) \mathrm{UA}\left(\mathrm{T}_{\mathrm{LC}}-\mathrm{T}_{\mathrm{L}}\right)
$$

Si por otra parte, se aplican la primera y la segunda leyes de la Termodinámica al ciclo de Carnot de la figura 1, se obtiene para la potencia de salida de la máquina,

$$
\dot{\mathrm{W}}=\frac{\dot{\mathrm{Q}}_{L C} \mathrm{~T}_{H C}}{\mathrm{~T}_{\mathrm{LC}}}-\dot{\mathrm{Q}}_{\mathrm{LC}}
$$

que combinada con la ecuación 6 , resulta en:

$$
\dot{W}=(1-x) \operatorname{UAT}_{\mathrm{L}}\left(\frac{\mathrm{T}_{\mathrm{LC}}}{\mathrm{T}_{\mathrm{L}}}-1\right)\left(\frac{\mathrm{T}_{H C}}{\mathrm{~T}_{\mathrm{LC}}}-1\right)
$$

Si además se definen

$$
\tau=\frac{T_{H}}{T_{L}}
$$

y

$$
\tau_{\mathrm{C}}=\frac{\mathrm{T}_{\mathrm{HC}}}{\mathrm{T}_{\mathrm{LC}}}
$$

se puede obtener una expresión

$$
\frac{\mathrm{T}_{\mathrm{LC}}}{\mathrm{T}_{\mathrm{L}}}=1-x+\chi \frac{\tau}{\tau_{\mathrm{C}}}
$$

que sustituida en la ecuación 8 , permite obtener finalmente:

$$
\dot{W}=x(1-x) \operatorname{UAT}_{L}\left(\frac{\tau}{\tau_{C}}-1\right)\left(\tau_{C}-1\right)
$$

\section{Potencia máxima}

La ecuación 11 se puede maximizar con respecto a la relación de temperaturas del ciclo reversible, $\tau_{c}$, o bien, con respecto a la razón de distribución de la conductancia $x$. El primer criterio da como resultado que

$$
\left(\frac{T_{H C}}{T_{L C}}\right)_{\text {opt }}=\left(\frac{T_{H}}{T_{L}}\right)^{1 / 2}
$$

es decir, las temperaturas del ciclo deben guardar cierta relación con las temperaturas de la fuente y el sumidero de calor con las cuales interactúa el ciclo.

El segundo criterio de maximización establece que

$$
(\mathrm{UA})_{\mathrm{H}}=(\mathrm{UA})_{\mathrm{L}}
$$

es decir, para obtener una potencia máxima de salida, las conductancias térmicas de los intercambiadores de calor en los extremos caliente y frío, deben ser iguales.

Teniendo en cuenta los resultados expresados mediante las ecuaciones 12 y 13 , se obtiene la potencia doblemente maximizada,

$$
\dot{\mathrm{W}}_{\text {max }, \text { max }}=\frac{1}{4} \operatorname{UAT}_{\mathrm{L}}\left(\tau^{1 / 2}-1\right)^{2}
$$

Se puede concluir de todo lo anterior que para lograr la máxima potencia, debe haber por una parte, un cierto ajuste entre las relaciones de temperatura, dado por la ecuación 12; y por otra parte, debe existir también un determinado balance en los tamaños de los intercambiadores de calor en los dos extremos de temperatura, de acuerdo con la ecuación 13.

\section{Eficiencias del modelo}

Bejan (1996a) propone una tercera vía para maximizar la potencia de salida de su modelo, 
mediante el compromiso entre las inversiones en el equipo total de intercambio térmico y en los materiales y accesorios que conformarían la resistencia térmica $\mathrm{R}_{i}$ a la transferencia de calor interna (Ec. 1, Fig. 1). Para ello, conviene recordar la eficiencia de la Segunda Ley, definida como

$$
\eta_{11}=\frac{\eta}{\eta_{c}}
$$

donde $\eta$ es la eficiencia de la primera ley y $\eta_{c}$ es la eficiencia de Carnot. Para el ciclo de la figura 1, con el trabajo doblemente maximizado, se tendrían:

$$
\eta=\frac{\dot{\mathrm{W}}_{\max , \max }}{\dot{\mathrm{Q}}_{\mathrm{H}}} \quad \eta_{c}=1-\frac{\mathrm{T}_{\mathrm{L}}}{\mathrm{T}_{\mathrm{H}}}
$$

Se obtiene así, después de algunos pasos algebraicos,

$$
\eta_{I I}=\left[\frac{4}{R_{i} U A}\left(1+\tau^{-1 / 2}\right)^{2}+1+\tau^{-1 / 2}\right]^{-1}
$$

La ecuación 17 muestra que $\eta_{\|}$sólo depende de dos parámetros, $\tau=\mathrm{T}_{\mathrm{H}} / \mathrm{T}_{\mathrm{L}}$ y el grupo adimensional $R_{i} U A$; y se puede ver que $\eta_{\|}$decrece conforme $R_{i} U A$ disminuye. Entonces idealmente convendría aumentar $R_{i}$ y UA para incrementar $\eta_{l l}$, pero lo anterior está limitado por las restricciones económicas. Se puede suponer que en el costo total de la central, tanto $\mathrm{R}_{i}$ como UA compiten entre sí, de tal manera que:

$$
\mathrm{P}_{c} \mathrm{UA}+\mathrm{P}_{r} \mathrm{R}_{i}=\mathrm{Cte}
$$

donde $\mathrm{P}_{c}$ y $\mathrm{P}_{r}$ son los costos unitarios asociados con incrementar conductancia térmica y resistencia térmica de aislamiento, respectivamente.

El autor del método, propone definir un parámetro de colocación de la inversión, mediante el cual se pueden optimar los recursos disponibles tanto para UA como para $R_{i}$. Se obtiene así, para la eficiencia máxima de la Segunda Ley,

$$
\eta_{l l \max }=\left[16 \pi\left(1+\tau^{-1 / 2}\right)^{2}+1+\tau^{-1 / 2}\right]^{-1}
$$

donde $\pi$ es el cociente adimensional de precios,

$$
\pi=\frac{P_{r}}{P_{c} C^{2}}
$$

Obsérvese en particular que $\pi=0$ representa el límite correspondiente a una disponibilidad infinitamente amplia de aislamiento térmico (o de cero fuga de calor), en cuyo caso, la ecuación 19 se reduce a:

$$
\eta_{l l}=\frac{\tau^{1 / 2}}{\tau^{1 / 2}+1}
$$

y teniendo en cuenta la ecuación 15, resulta

$$
\eta=\eta_{C A}=1-\tau^{-1 / 2}=1-\left(\frac{T_{L}}{T_{H}}\right)^{1 / 2}
$$

En esta última ecuación, la eficiencia $\eta$ es la misma que en los modelos de centrales optimadas por Novikov (1958), Chambadal (1958) y Curzon y Ahlborn (1975).

\section{Ejemplos}

En la figura 2 se ha graficado la ecuación 19 para diferentes valores del parámetro $\pi$, y superpuestas en la gráfica se muestran las eficiencias de la Segunda Ley, ecuación 15, de diez centrales termoeléctricas en diferentes partes del mundo junto con otras diez centrales mexicanas, basadas en los parámetros de operación reales que se indican en las tablas 1 y 2. Para las primeras se aprovecharon los datos apuntados por Spalding y Cole (1973), que han sido repetidamente utilizados en la literatura; para las segundas se recabó la información de las fuentes disponibles en México.

Es necesario señalar, que tanto en las centrales mexicanas como en las de otras partes del mundo, se ha tomado para la temperatura de la fuente de calor ( $\mathrm{T}_{\mathrm{H}}$, tercera columna de las tablas 1 y 2$)$, el valor de la temperatura de la sustancia de trabajo en el interior del ciclo de Rankine, por no disponerse en ningún caso (probablemente ni se conozca), del valor real de la temperatura de dicha fuente, que en la realidad debe ser un valor cercano a la temperatura de los gases en combustión. Esto induce a pensar erróneamente que las centrales operan con altas eficiencias de la Segunda Ley y que 
DOI: http://dx.doi.org/10.22201/fi.25940732e.2002.03n1.001

Tabla 2. Eficiencias de varias centrales mexicanas calculadas en el presente trabajo. (Datos de la Comisión Federal de Electricidad).

\begin{tabular}{|c|c|c|c|c|c|c|c|}
\hline CENTRAL & $\mathrm{T}_{\mathrm{L}}\left({ }^{\circ} \mathrm{C}\right)$ & $\mathrm{T}_{\mathrm{H}}\left({ }^{\circ} \mathrm{C}\right)$ & $\tau$ (Ec. 9a) & $\eta_{c}$ & $\eta_{C A}(E C .22)$ & $\begin{array}{c}\eta \\
\text { (observada) }\end{array}$ & $\eta_{I I}($ Ec. 21$)$ \\
\hline Tuxpan & 27 & 542 & 2.71 & 0.63 & 0.39 & 0.38 & 0.60 \\
\hline Tula & 23 & 542 & 2.75 & 0.63 & 0.39 & 0.38 & 0.59 \\
\hline Valle de México & 22 & 541 & 2.76 & 0.63 & 0.39 & 0.37 & 0.58 \\
\hline Punta prieta & 28 & 513 & 2.61 & 0.61 & 0.38 & 0.35 & 0.56 \\
\hline Monterrey & 28 & 541 & 2.70 & 0.63 & 0.39 & 0.37 & 0.59 \\
\hline Manzanillo & 28 & 537 & 2.69 & 0.62 & 0.39 & 0.37 & 0.58 \\
\hline Mazatlán & 28 & 537 & 2.69 & 0.62 & 0.39 & 0.32 & 0.50 \\
\hline San Luis Potosí & 25 & 538 & 2.72 & 0.63 & 0.39 & 0.35 & 0.55 \\
\hline Lerma & 25 & 510 & 2.62 & 0.61 & 0.38 & 0.36 & 0.59 \\
\hline
\end{tabular}

\section{Conclusiones}

Se han presentado los elementos de la optimación de los ciclos convencionales de centrales termoeléctricas, atendiendo a criterios derivados de la consideración moderna de la segunda ley de la Termodinámica. El análisis permite replantear aspectos poco conocidos acerca de las centrales en operación y ofrece oportunidades mejoradas que conviene tener en cuenta para el diseño de nuevas centrales.

\section{Agradecimientos}

Se agradece la colaboración de Rocío Pedroza y Mauricio de Alba del Posgrado de Ingeniería de la UNAM, así como a Víctor Esparza del LAPEM de la CFE, quienes proporcionaron datos de las centrales mexicanas.

\section{Referencias}

Bejan A. (1988). Advanced Engineering Thermodynamics. Wiley, New York.

Bejan A. (1996a). Entropy Generation Minimization. CRC Press, Boca Raton.

Bejan A. (1996b). Models of Power Plants that Generate Minimum Entropy while Operating at Maximum Power. Am. J. Phys., Vol. 64, 1054-1059.
Bejan A.H. y Paynter H.M. (1976). Solved Problems in Thermodynamics. MIT, Cambridge.

Bejan A., Tsatsaronis G. y Moran M.J. (1996). Thermal Design and Optimization. Wiley, New York.

Bejan A. y Mamut E. (1998). Thermodynamic Optimization of Complex Energy Systems. NATO Science Series, 3. High Technology, Vol. 69, Kluwer Academic Publishers, London.

Chambadal P. (1958). Le Choix Du Cycle Thermique Dans Une Usine Generatrice Nucleaire. Rev. Gen. Electr., Vol. 67, 332-345.

Curzon F.L. y Ahlborn B. (1975). Efficiency of a Carnot Engine at Maximum Power Output. Am. J. Phys., Vol. 43, 22-24.

El-Wakil M.M (1984). PowerPlant Technology. McGraw-Hill Book Co., New York.

Novikov I.I. (1958). The Efficiency of Atomic Power Plants. J. Nuclear Engrg. II, Vol. 7, 125-128.

Salamon P., Hoffmann K.H., Schubert S., Berry S. y Andresen B. (2001). What Conditions Make Minimum Entropy Production Equivalent to Maximum Power Production? J. Non-Equilib. Thermodyn., Vol. 26, 73-83.

Spalding D.B. y Cole E.H. (1973). Engineering Thermodynamics. $3^{\text {rd }}$. Edward Arnold, London.

Wark Jr. K. (1998). Termodinámica. 5a. Ed. McGraw-Hill, México. 
DOI: http://dx.doi.org/10.22201/fi.25940732e.2002.03n1.001

J. Cervantes-de Gortari, A.Vidal-Santo, F. Méndez-Lavielle y O. Bautista-Godinez

\section{Semblanza de los autores}

Jaime Cervantes-de Gortari. Concluye su carrera como ingeniero mecánico en la Facultad de Ingeniería de la UNAM en 1970 , obtuvo su maestría en ingeniería mecánica el mismo año y se doctoró en ingeniería mecánica en Purdue University en 1976. Actualmente es profesor de tiempo completo y catedrático UNAM de la Facultad de Ingeniería de la UNAM.

Adrián Vidal-Santo. Se gradúa como ingeniero mecánico electricista en la Universidad Juárez Autónoma de Tabasco en 1999 y actualmente es candidato al grado de maestro en ingeniería mecánica en la UNAM.

Federico Méndez-Lavielle. Físico egresado de la UNAM en 1980, cursa su maestría en ingeniería mecánica en la Facultad de Ingeniería, UNAM en 1986. Obtuvo su doctorado en ingeniería mecánica en la misma institución en 1992. Actualmente es profesor de tiempo completo y catedrático UNAM de la Facultad de Ingeniería de la UNAM.

Oscar Bautista-Godínez. Finaliza la carrera de ingeniero mecánico en la Facultad de Ingeniería, UNAM en 1995, obtuvo su maestría en ingeniería mecánica en 1998 y actualmente es candidato a doctor en ingeniería mecánica de la UNAM. Asimismo, es profesor de tiempo completo en el ITESM, campus Ciudad de México. 
DOI: http://dx.doi.org/10.22201/fi.25940732e.2002.03n1.001 